\title{
Patients Undergoing Primary Percutaneous Coronary Intervention
}

\author{
Yacov Shacham, MD; Eran Leshem-Rubinow, MD; Amir Gal-Oz, MD; \\ Eyal Ben-Assa, MD; Arie Steinvil, MD; Gad Keren, MD; Arie Roth, MD; Yaron Arbel, MD \\ Department of Cardiology (Shacham, Leshem-Rubinow, Ben-Assa, Steinvil, Keren, Roth, Arbel) \\ and Department of Nephrology (Gal-Oz), Tel-Aviv Sourasky Medical Center affiliated to the \\ Sackler Faculty of Medicine, Tel-Aviv University, Tel-Aviv, Israel
}

Background: The worsening of serum creatinine $(\mathrm{s} C r$ ) level is a frequent finding among ST-segment elevation MI (STEMI) patients undergoing primary percutaneous coronary intervention $(\mathrm{PCI})$, associated with adverse short-term and long-term outcomes. No information is present, however, regarding the incidence and prognostic implications associated with an improvement in sCr levels throughout hospitalization, as compared with admission levels.

Hypothesis: Reversible renal impairment prior to $\mathrm{PCl}$ is not associated with adverse outcomes.

Methods: We retrospectively studied 1260 STEMI patients undergoing primary $\mathrm{PCI}$. The incidence of in-hospital complications and long-term mortality was compared between patients having stable, worsened $(>0.3 \mathrm{mg} / \mathrm{dL}$ increase), or improved ( $>0.3 \mathrm{mg} / \mathrm{dL}$ decrease) $\mathrm{sCr}$ levels throughout hospitalization.

Results: Overall, 127 patients (10\%) had worsening in sCr levels, whereas $44(3.5 \%)$ had an improvement of sCr compared with admission levels. Patients with worsening SCR had more complications during hospitalization, higher 30 -day ( $13 \%$ vs $1 \% ; P<0.001)$ and up to 5 -year all-cause mortality $(28 \%$ vs $5 \% ; P<0.001)$ compared with those with stable SCR. No significant difference was found regarding complications and mortality between patients having an improvement in $\mathrm{s} C r$ and stable $\mathrm{s} C \mathrm{r}$. Compared with patients with stable $\mathrm{s} C \mathrm{r}$, the adjusted hazard ratio for all-cause mortality in patients with worsened $\mathrm{s} C \mathrm{C}$ was 6.68 (95\% confidence interval: 2.1-21.6, $P=0.002$ ).

Conclusions: In STEMI patients undergoing primary $\mathrm{PCl}$, renal impairment prior to $\mathrm{PCl}$ is a frequent finding. In contrast to post- $\mathrm{PCl} \mathrm{sCr}$ worsening, this entity is not associated with adverse short-term and long-term outcomes.

\section{Introduction}

Acute kidney injury (AKI) frequently complicates the course of acute ST-segment elevation myocardial infarction (STEMI) and is associated with adverse outcomes. ${ }^{1-4}$ The worsening of renal function throughout hospitalization in STEMI patients is multifactorial, though the most important reason is considered contrast-induced nephropathy, related mainly to the amount of contrast material and to preprocedural renal function. ${ }^{5-7}$ Additional important

All authors take responsibility for all aspects of the reliability and freedom from bias of the data presented and their discussed interpretation.

The authors have no funding, financial relationships, or conflicts of interest to disclose. reasons include hemodynamic state, drugs admitted (especially blockers of the renin-angiotensin axis), as well as the occurrence of sepsis, bleeding, atheroembolic disease, and acute hyperglycemia. ${ }^{8-10}$ Although all trials assessing AKI among STEMI patients defined this complication on the basis of the rise of serum creatinine $(\mathrm{sCr})$ during hospitalization compared with admission sCr levels, ${ }^{1-4,11,12}$ no trial to date has examined the presence of rapid reversal of renal impairment present at hospital admission, prior to PCI, and its prognostic effect among STEMI patients. In the present study, we compared the incidence, inhospital complications, as well as the short-term and long-term mortality associated with $\mathrm{sCr}$ change patterns in a large cohort of consecutive STEMI patients undergoing primary PCI.
274. Clin. Cardiol. 38, 5, 274-279 (2015)

Published online in Wiley Online Library (wileyonlinelibrary.com) DOI:10.1002/clc.22384 ( 2015 Wiley Periodicals, Inc.
Received: November 14, 2014

Accepted with revision: December 17, 2014 


\section{Methods}

\section{Study Population}

We performed a retrospective, single-center observational study at the Tel-Aviv Sourasky Medical Center, a tertiary referral hospital with a $24 / 7$ primary PCI service, including all 1367 consecutive patients admitted between January 2008 and July 2013 to the cardiac intensive care unit with the diagnosis of acute STEMI. Excluded were 28 patients who were treated either conservatively or with thrombolysis and 63 patients whose final diagnosis on discharge was other than anterior-wall STEMI (eg, myocarditis or takotsubo cardiomyopathy). Also excluded were patients who died within 24 hours of admission $(n=12)$, because they would not have had sufficient time to develop post-PCI AKI, and patients requiring chronic peritoneal or hemodialysis treatment $(n=4)$. The final study population included 1260 patients whose baseline demographics, cardiovascular history, clinical risk factors, treatment characteristics, and laboratory results were retrieved from their medical files.

\section{Protocol}

The diagnosis of STEMI was established by a typical history of chest pain, diagnostic electrocardiographic changes, and serial elevation of serum cardiac biomarkers. ${ }^{13}$ Blood samples were drawn during admission of all patients. Primary PCI was performed in patients with symptoms $\leq 12$ hours in duration, as well as in patients with symptoms lasting 12 to 24 hours in duration if the symptoms continued to persist at the time of admission. Patient records were evaluated for in-hospital mortality and complications occurring during the hospitalization. These included cardiogenic shock or the need for intra-aortic balloon counterpulsation treatment, need for emergent coronary artery bypass graft surgery, mechanical ventilation or heart failure episodes treated conservatively, clinically significant tachyarrhythmias, bradyarrhythmias requiring pacemaker, as well as major bleeding (requiring blood transfusion). Mortality was assessed over a median period of $1526 \pm 298$ days (range, 2-2130 days) up to August 1, 2013. Assessment of survival following hospital discharge was determined from computerized records of the population registry bureau. The study protocol was approved by the local institutional ethics committee.

\section{Laboratory Parameters}

The $\mathrm{sCr}$ was determined upon hospital admission and at least once a day during the cardiac intensive care unit stay and was available for all analyzed patients. The estimated glomerular filtration rate (eGFR) was estimated using the abbreviated Modification of Diet in Renal Disease (MDRD) equation. ${ }^{14}$ Baseline renal insufficiency was categorized as an eGFR $\leq 60 \mathrm{~mL} / \mathrm{min} / 1.73 \mathrm{~m}^{2} .{ }^{15}$ Patients were stratified into 3 groups according their $\mathrm{s} C r$ change patterns, compared with $\mathrm{sCr}$ levels at hospital admission, using the Acute Kidney Injury Network (AKIN) criteria ${ }^{16}$ : stable $\mathrm{sCr}(<0.3 \mathrm{mg} / \mathrm{dL}$ change), worsened $\mathrm{sCr}$ (defined as an increase in $\mathrm{sCr}$ $>0.3 \mathrm{mg} / \mathrm{dL}$ within 48 hours of admission), and improved $\mathrm{sCr}$ (defined as both $\mathrm{sCr}>1.2 \mathrm{mg} / \mathrm{dL}$ upon hospital admission and concomitant decrease of $>0.3 \mathrm{mg} / \mathrm{dL}$ in
sCr within 48 hours). When available, sCr upon hospital discharge was compared with follow-up sCr in patients with AKI. Renal function recovery was defined as maintained sCr levels (>30 days) compared with the hospital discharge levels.

\section{Statistical Analysis}

All data were summarized and displayed as mean \pm standard deviation or median $(25 \%-75 \%)$ for continuous variables and as number of patients (\%) in each group for categorical variables. The $P$ values for the $\chi^{2}$ square test were calculated with the Fisher exact test. Continuous variables were compared using the independent sample $t$ test or Mann-Whitney test. The identification of the independent predictors of AKI was assessed using logistic regression. The influence of $\mathrm{sCr}$ change on the occurrence of all-cause mortality was evaluated using multivariate Cox regression, adjusted for age, sex, hypertension, diabetes mellitus, left ventricular ejection fraction, eGFR, baseline hemoglobin, white blood cell count, high-sensitivity C-reactive protein (hs-CRP), and AKIN status. A 2-tailed $P$ value of $<0.05$ was considered significant for all analyses. All analyses were performed with SPSS version 21 software (IBM Corp., Armonk, NY).

\section{Results}

A total of 1260 STEMI patients treated by primary PCI were enrolled in the study, 294 (23\%) of whom had baseline renal insufficiency upon hospital admission. A total of 127 patients $(10 \%)$ had worsening of $\mathrm{sCr}$, whereas 44 patients (3.5\%) had an improvement in sCr. Only 2 (1\%) patients among those developing AKI required renal-replacement therapy throughout hospitalization; both had worsened $\mathrm{sCr}$ following PCI.

The baseline clinical characteristics of patients with stable, improved, and worsened $\mathrm{sCr}$ are listed in Table 1. With the exception of diabetes mellitus and admission hsCRP levels, no significant differences were present between patients with stable and improved $\mathrm{sCr}$. On the other hand, patients with worsened $\mathrm{sCr}$ were more likely to be older and female and to have more comorbidities, longer symptom duration prior to emergency room admission, advanced coronary artery disease, higher hs-CRP, lower left ventricular ejection fraction, and longer time until hospital discharge.

The worsening of sCr following STEMI resulted in more complications and adverse events during hospitalization, as well as higher 30-day mortality. Those findings, however, were not found in patients with improved $\mathrm{sCr}$ (Table 2). Follow-up sCr was available in 33 of 44 of patients with improved sCr, with 30 of 33 patients demonstrating maintained recovery of renal function.

\section{Long-term Outcome}

Over a mean period of $1526 \pm 298$ days (range, 2-2130 days), 95 (7.5\%) patients of the entire cohort died. Mortality was significantly higher among those with worsening of sCr post-PCI AKI (35 of 127, 28\%) following STEMI than those with stable sCr (55 of 1089, 5.1\%; $P<0.001$ ) and those 
Table 1. Baseline Characteristics of 1260 STEMI Patients According to SCR Change Pattern

\begin{tabular}{|c|c|c|c|c|c|}
\hline Variable & $\begin{array}{c}\text { sCR Stable, } \\
n=1089\end{array}$ & $\begin{array}{c}\text { sCR Improved, } \\
n=44\end{array}$ & $P$ Value & $\begin{array}{c}\text { sCr Worsened, } \\
n=127\end{array}$ & $P$ Value $^{a}$ \\
\hline Age, $y$ & $60 \pm 12$ & $64 \pm 14$ & 0.05 & $72 \pm 12$ & $<0.001$ \\
\hline Male sex & $891(82)$ & $34(77)$ & 0.43 & 88 (69) & 0.003 \\
\hline DM & 211 (19) & $15(34)$ & 0.02 & 39 (31) & 0.001 \\
\hline Dyslipidemia & $502(46)$ & $23(52)$ & 0.44 & $69(54)$ & 0.167 \\
\hline Hypertension & $441(41)$ & $22(50)$ & 0.21 & $91(72)$ & $<0.001$ \\
\hline Smoking history & $568(52)$ & $20(45)$ & 0.44 & $43(34)$ & $<0.001$ \\
\hline Family history of CAD & $183(17)$ & $7(16)$ & 0.99 & $8(6)$ & 0.009 \\
\hline Prior MI & 103 (9) & $2(4)$ & 0.42 & 14 (11) & 0.49 \\
\hline \multicolumn{6}{|l|}{ No. of narrowed coronary arteries } \\
\hline 1 & 489 (45) & $15(34)$ & 0.49 & $46(36)$ & 0.07 \\
\hline 2 & $327(30)$ & 17 (39) & 0.47 & $34(27)$ & 0.07 \\
\hline 3 & $268(25)$ & $12(27)$ & 0.26 & $47(37)$ & 0.003 \\
\hline Time to ED, $\min$ & $374 \pm 659$ & $420 \pm 808$ & 0.39 & $528 \pm 577$ & $<0.001$ \\
\hline D2B time, $\min$ & $44 \pm 40$ & $44 \pm 19$ & 0.87 & $45 \pm 19$ & 0.24 \\
\hline Contrast material amount, $\mathrm{mL}^{b}$ & $149 \pm 18$ & $134 \pm 17$ & 0.359 & $128 \pm 52$ & 0.06 \\
\hline Admission eGFR, $\mathrm{mL} / \mathrm{min} / 1.73 \mathrm{~m}^{2}$ & $73 \pm 17$ & $50 \pm 14$ & $<0.001$ & $55 \pm 20$ & $<0.001$ \\
\hline Admission sCr, mg/dL & $1.11 \pm 0.19$ & $1.60 \pm 0.40$ & $<0.001$ & $1.34 \pm 0.44$ & $<0.001$ \\
\hline Peak sCr, mg/dL & $1.14 \pm 0.20$ & $1.60 \pm 0.40$ & $<0.001$ & $2.08 \pm 0.97$ & $<0.001$ \\
\hline sCr change in hospital, mg/dL & $0.03 \pm 0.17$ & $-0.40 \pm 0.15$ & $<0.001$ & $0.65 \pm 0.69$ & $<0.001$ \\
\hline $\mathrm{sCr}$ at discharge, $\mathrm{mg} / \mathrm{dL}$ & $1.07 \pm 0.17$ & $1.19 \pm 0.30$ & 0.007 & $1.64 \pm 0.77$ & $<0.001$ \\
\hline Duration of hospitalization, $d$ & $5.3 \pm 2.9$ & $7.0 \pm 5.7$ & 0.11 & $9.4 \pm 7.6$ & $<0.001$ \\
\hline Peak CPK, U/L & $1180 \pm 1384$ & $1053 \pm 1135$ & 0.84 & $1231 \pm 1438$ & 0.93 \\
\hline Admission CRP, mg/dL & $11.3 \pm 25.3$ & $29.7 \pm 54$ & 0.008 & $25.8 \pm 43.8$ & $<0.001$ \\
\hline LVEF, \% & $48 \pm 8$ & $48 \pm 11$ & 0.70 & $43 \pm 9$ & $<0.001$ \\
\hline
\end{tabular}

Abbreviations: CAD, coronary artery disease; CPK, creatine phosphokinase; CRP, C-reactive protein; D2B, door-to-balloon; DM, diabetes mellitus; ED, emergency department; eGFR, estimated glomerular filtration rate; LVEF, left ventricular ejection fraction; MI, myocardial infarction; sCR, serum creatinine; SD, standard deviation.

Data are presented as mean \pm SD or $\mathrm{n}(\%)$.

${ }^{a} P$ value for worsened sCR vs stable $\mathrm{sCr}$. ${ }^{b}$ Information on contrast volume amount was present for only 418 patients.

with improved $\mathrm{sCr}$ (5 of 44, 11\%; $P<0.001$; Figure 1). In multivariate analysis, worsening of $\mathrm{sCr}$ was an independent predictor of mortality, reaching a hazard ratio of $6.2(95 \%$ confidence interval: 2.2-17.3; $P<0.001$ ), compared with patients with stable $\mathrm{sCr}$. Improved $\mathrm{sCr}$ was not associated with increased risk for long-term mortality (hazard ratio: $0.17,95 \%$ confidence interval: $0.008-3.4, P=0.24$ ).

\section{Discussion}

This is the first study comparing the patterns of $\mathrm{sCr}$ changes among STEMI patients undergoing primary PCI and their relation to the angiographic procedure and coronary intervention. We found that unlike post-PCI AKI, rapid reversal of AKI present upon hospital admission is a less frequent complication and has no major adverse effects on short-term and long-term outcomes. Moreover, the majority of patients with an improvement of $\mathrm{sCr}$ had maintained recovery of their renal function over a 30-day follow-up period.

Contrast material is still considered the major reason for AKI development in STEMI patients following PCI. Contrast-induced acute kidney injury (CI-AKI) is a prevalent and deleterious complication of coronary angiography and reported to be the third most common cause of hospitalacquired renal failure. ${ }^{5}$ The risk of CI-AKI is directly associated with increasing contrast-media volume, ${ }^{6}$ with 
Table 2. In-hospital Complications of 1260 STEMI Patients According sCr Change Pattern

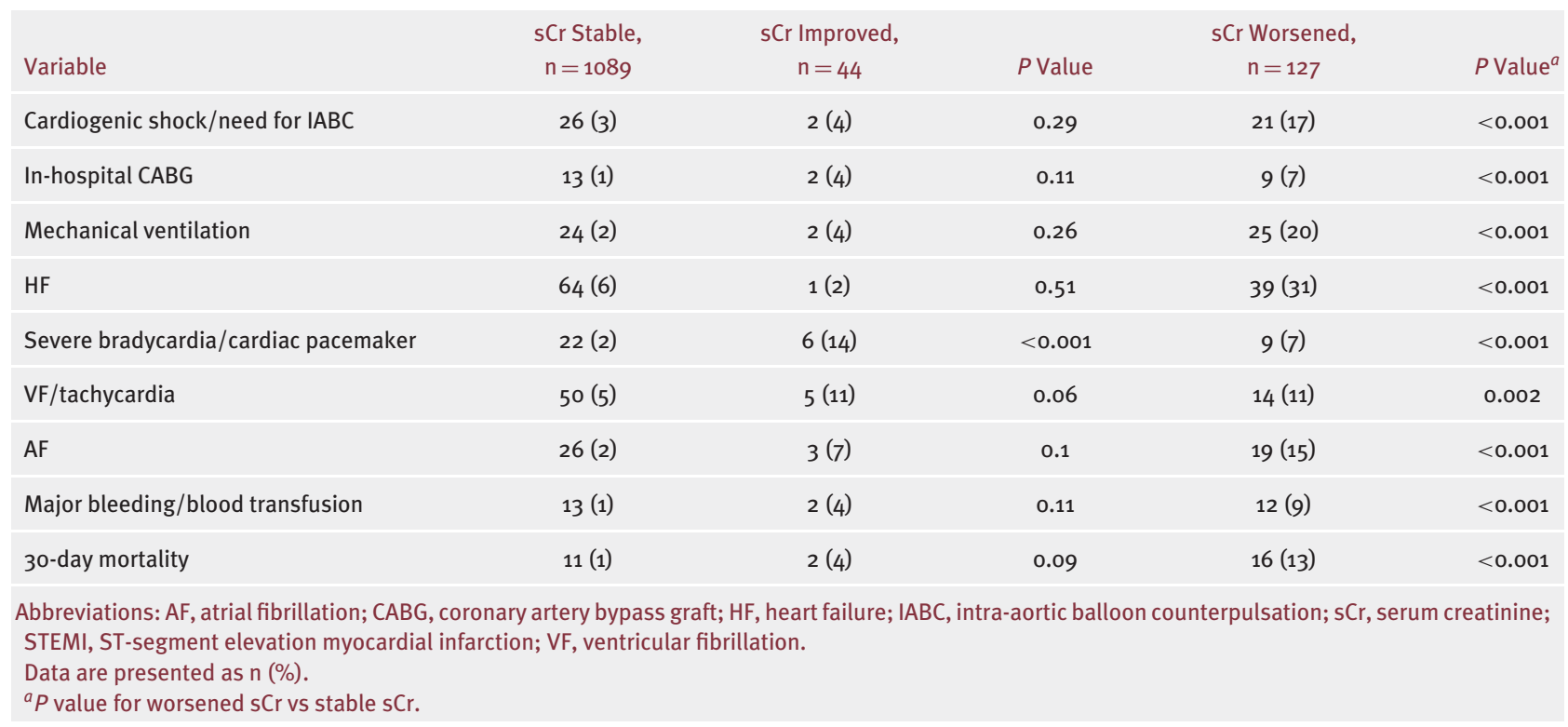

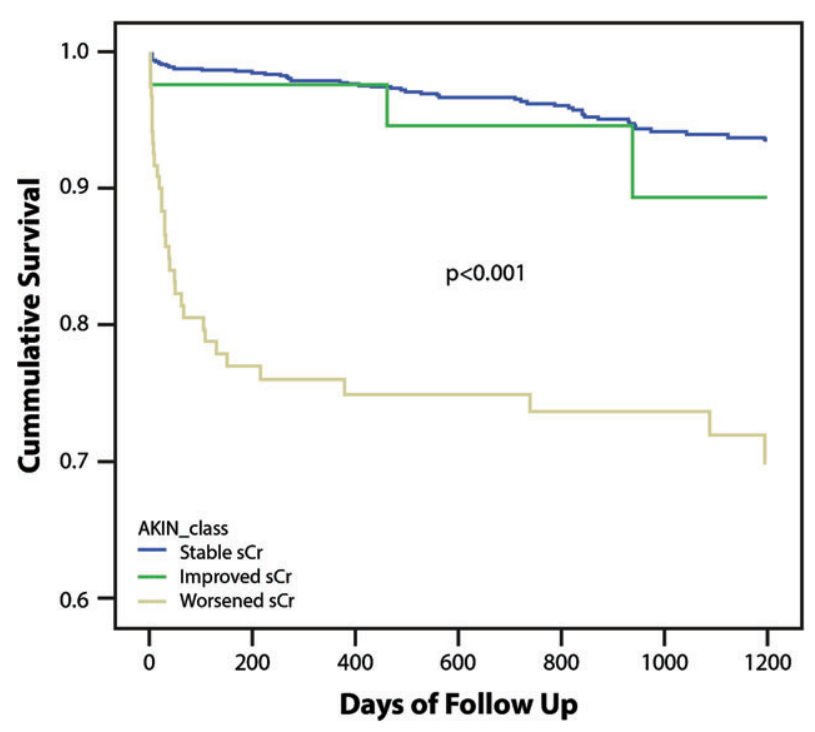

Figure 1. Cumulative survival rates for 1260 patients with STEMI on the basis of $\mathrm{s} C r$ changes throughout hospitalization $(P<0.001$ for patients with stable sCr or improved sCr vs worsened sCr). Abbreviations: AKIN, Acute Kidney Injury Network; sCR, serum creatinine; STEMI, ST-segment elevation myocardial infarction.

incidence ranging from $2 \%$ among patients with normal baseline renal function to as high as $20 \%$ to $30 \%$ in patients with a chronic renal failure. ${ }^{17}$ Even after adjusting for baseline renal function and comorbidities, in-hospital mortality is about 5-fold higher in CI-AKI patients, and longterm mortality rates are about 4 -fold higher. ${ }^{7}$ It is important to state that in our report patients developing post-PCI AKI received less contrast material during $\mathrm{PCI}$, probably due to the elevated $\mathrm{sCr}$ levels found upon admission in that group. This finding and the demonstration of renal impairment prior to PCI and contrast injection implies that other factors aside from CI-AKI contribute to AKI development in the early setting of STEMI. The sudden myocardial insult in STEMI often results in an acute reduction of cardiac output, and this early hemodynamic deterioration may theoretically lead to reduced renal perfusion and early worsening of renal function. Short-term renal hypoperfusion is often associated with a prerenal failure, defined as a reversible loss of renal function without structural damage. ${ }^{18}$ A more profound and prolonged hypoperfusion primarily affects the function and structure of tubular epithelial cells, which, in severe cases, is characterized by epithelial-cell ischemia and necrosis.

In accordance with a previous report by our group, ${ }^{1}$ patients developing post-PCI AKI had longer symptom duration, probably resulting in worse left ventricular function, with prolonged impairment of hemodynamics and renal perfusion. Symptom duration is a powerful prognostic marker in STEMI patients undergoing reperfusion, ${ }^{19,20}$ and major consideration is given to minimizing ischemic duration to improve survival following STEMI. ${ }^{21}$ Longer ischemia duration is associated with more extensive and possibly irreversible myocardial damage, ${ }^{22}$ resulting in longer renal hypoperfusion, which may convert early prerenal insult into a renal (tubular or glomerular) injury.

There are currently only limited reports regarding the timing of the renal insult and its relation to clinical outcome. Kim et al reported that transient and persistent moderate/severe AKI during acute myocardial infarction is strongly related to 1-year all-cause mortality after STEMI. ${ }^{23}$ An additional study evaluated the impact of transient and persistent acute kidney injury on long-term outcomes after STEMI, ${ }^{24}$ revealing that even transient AKI in these patients portends increased long-term mortality and that patients with persistent AKI are in the highest risk group. Those studies defined transient AKI as transient worsening and improvement of $\mathrm{sCr}$ throughout hospitalization, as compared with admission sCr levels, and primary PCI was not applied to all patients. Contrary to these reports, 
we defined the improved $\mathrm{sCr}$ group as a continuous and persistent decline compared with admission levels, as was reported by Tian et al. ${ }^{25}$ Their results demonstrated that an increase in $\mathrm{sCr}$ level during hospitalization predicted worse outcomes, even if the $\mathrm{sCr}$ value returns to normal, whereas patients who presented to the hospital with an increased $\mathrm{sCr}$ level that returned rapidly to normal had outcomes approaching those of patients with $\mathrm{sCr}$ levels consistently in the normal range. We believe that the elevated $\mathrm{sCr}$ on admission with improving AKI represents an early, reversible hemodynamic-induced kidney injury or, alternatively, changes in nonrenal $\mathrm{Cr}$ kinetics (eg, $\mathrm{Cr}$ production during illness).

Our findings carry some important clinical implications. As we have shown, the baseline $\mathrm{sCr}$ value early after admission for STEMI may not reflect the true baseline sCr, especially if it is mild and no history of risk factors or prior renal failure is present. The utilization of novel markers of tubular injury such as urinary/plasma neutrophil gelatinaseassociated lipocalin, urinary kidney injury molecule-1, and urinary interleukin- $18^{26,27}$ offer the opportunity to diagnose AKI proactively and may help to differentiate those in whom mild $\mathrm{sCr}$ represents an acute response rather than a chronic finding, allowing early interventions for renal protection.

\section{Study Limitations}

Our study bears some limitations. Data regarding the amount of fluids given to patients were absent in the majority of patients; thus, their possible effect on renal function recovery could not be determined. Similarly, concomitant therapy data with statins, renin-angiotensin blockers, and diuretics throughout hospitalization were not present for many patients, and their effect on AKI development could not be assessed.

Although the AKI definition using the AKIN criteria ${ }^{16}$ refers to a $\mathrm{sCr}$ increase compared with the baseline value, the $\mathrm{sCr}$ at hospital admission, as we demonstrated, may not represent a true baseline value in STEMI patients, and follow-up sCr was not available for most patients with no AKI. Finally, the definition of AKIN refers to $\mathrm{sCr}$ change within a time frame of 48 hours. As the change in $\mathrm{sCr}$ can lag beyond this time period due to delayed effects of contrast material and drugs, worsening of renal function might have occurred following hospital discharge in some patients; thus, the true incidence of AKI described in our study may have been an underestimation. Finally, as retrospective data regarding baseline renal function were not available for most of the present cohort, it is not possible to exclude that those having only transient elevation of $\mathrm{sCr}$ were more likely to have normal function at baseline compared with those with worsened $\mathrm{sCr}$.

\section{Conclusion}

The worsening of renal function prior to PCI in STEMI patients is a frequent finding. In contrast to the welldescribed post-PCI AKI, this entity is often completely reversible and not associated with adverse short-term and long-term outcomes.

\section{References}

1. Shacham Y, Leshem-Rubinow E, Steinvil A, et al. Renal impairment according to Acute Kidney Injury Network criteria among STelevation myocardial infarction patients undergoing primary percutaneous intervention: a retrospective observational study. Clin Res Cardiol. 2014;103:525-532.

2. Goldberg A, Hammerman H, Petcherski S, et al. Inhospital and 1-year mortality of patients who develop worsening renal function following acute ST-elevation myocardial infarction. Am Heart J. 2005;150:330-337.

3. Parikh CR, Coca SG, Wang Y, et al. Long-term prognosis of acute kidney injury after acute myocardial infarction. Arch Intern Med. 2008;168:987-995.

4. Amin AP, Spertus JA, Reid KJ, et al. The prognostic importance of worsening renal function during an acute myocardial infarction on long-term mortality. Am Heart J. 2010;160:1065-1071.

5. James MT, Ghali WA, Knudtson ML, et al. Associations between acute kidney injury and cardiovascular and renal outcomes after coronary angiography. Circulation. 2011;123:409-416.

6. Gurm HS, Dixon SR, Smith DE, et al. Renal function-based contrast dosing to define safe limits of radiographic contrast media in patients undergoing percutaneous coronary interventions. $J \mathrm{Am}$ Coll Cardiol. 2011;58:907-914.

7. Seeliger E, Sendeski M, Rihal CS, et al. Contrast-induced kidney injury: mechanisms, risk factors, and prevention. Eur Heart J. 2012;33:2007-2015

8. Koreny M, Karth GD, Geppert A, et al. Prognosis of patients who develop acute renal failure during the first 24 hours of cardiogenic shock after myocardial infarction. Am J Med. 2002;112:115-119.

9. Marenzi G, Assanelli E, Campodonico J, et al. Contrast volume during primary percutaneous coronary intervention and subsequent contrast-induced nephropathy and mortality. Ann Intern Med. 2009;150:170-177.

10. Marenzi G, De Metrio M, Rubino M, et al. Acute hyperglycemia and contrast-induced nephropathy in primary percutaneous coronary intervention. Am Heart J. 2010;160:1170-1177.

11. Hwang SH, Jeong MH, Ahmed K, et al. Different clinical outcomes of acute kidney injury according to Acute Kidney Injury Network criteria in patients between ST-elevation and non-ST-elevation myocardial infarction. Int J Cardiol. 2011;150:99-101.

12. Marenzi G, Cabiati A, Bertoli SV, et al. Incidence and relevance of acute kidney injury in patients hospitalized with acute coronary syndromes. Am J Cardiol. 2013;111:816-822.

13. O'Gara PT, Kushner FG, Ascheim DD, et al. 2013 ACCF/AHA guideline for the management of ST-elevation myocardial infarction: a report of the American College of Cardiology Foundation/American Heart Association Task Force on Practice Guidelines. J Am Coll Cardiol. 2012;61:e78-e140.

14. Levey AS, Bosch JP, Lewis JB, et al; Modification of Diet in Renal Disease Study Group. A more accurate method to estimate glomerular filtration rate from serum creatinine: a new prediction equation. Ann Intern Med. 1999;130:461-470.

15. National Kidney Foundation (NKF) Kidney Disease Outcome Quality Initiative (K/DOQI) Advisory Board. K/DOQI clinical practice guidelines for chronic kidney disease: evaluation, classification, and stratification. Am J Kidney Dis. 2002;39:S1-S266.

16. Levin A, Warnock DG, Mehta RL, et al; Acute Kidney Injury Network Working Group. Improving outcomes from acute kidney injury: report of an initiative. Am J Kidney Dis. 2007;50:1-4.

17. Tehrani S, Laing C, Yellon DM, et al. Contrast-induced acute kidney injury following PCI. Eur J Clin Invest. 2013;43:483-490.

18. Devarajan P. Update on mechanisms of ischemic acute kidney injury. J Am Soc Nephrol. 2006;17:1503-1520.

19. Newby LK, Rutsch WR, Califf RM, et al; GUSTO-1 Investigators. Time from symptom onset to treatment and outcomes after thrombolytic therapy. J Am Coll Cardiol. 1996;27:1646-1655.

20. Cannon CP, Gibson CM, Lambrew CT, et al. Relationship of symptom-onset-to-balloon time and door-to-balloon time with mortality in patients undergoing angioplasty for acute myocardial infarction. JAMA. 2000;283:2941-2947.

21. Bradley EH, Nallamothu BK, Herrin JT, et al. National efforts to improve door-to-balloon time: results from the Door-to-Balloon Alliance. J Am Coll Cardiol. 2009;54:2423-2429. 
22. Reimer KA, Lowe JE, Rasmussen MM, et al. The wavefront phenomenon of ischemic cell death. 1. Myocardial infarct size vs duration of coronary occlusion in dogs. Circulation. 1977;56:786-794.

23. Kim MJ, Choi HS, Oh SH, et al. Rapid reversal of acute kidney injury and hospital outcomes: a retrospective cohort study. Yonsei Med J. 2011;52:603-609.

24. Goldberg A, Kogan E, Hammerman H, et al. The impact of transient and persistent acute kidney injury on long-term outcomes after acute myocardial infarction. Kidney Int. 2009;76:900-906.
25. Tian J, Barrantes F, Amoateng-Adjepong Y, et al. Rapid reversal of acute kidney injury and hospital outcomes: a retrospective cohort study. Am J Kidney Dis. 2009;53: 974-981.

26. Coca SG, Yalavarthy R, Concato J, et al. Biomarkers for the diagnosis and risk stratification of acute kidney injury: a systematic review. Kidney Int. 2008;73:1008-1016.

27. Koyner JL, Parikh CR. Clinical utility of biomarkers of AKI in cardiac surgery and critical illness. Clin J Am Soc Nephrol. 2013;8:1034-1042. 